\title{
Écartement et gabarit : la lente et difficile émergence de normes fondamentales
}

Clive Lamming

\section{(2) OpenEdition \\ Journals}

Édition électronique

URL : https://journals.openedition.org/rhcf/1939

DOI : 10.4000/rhcf.1939

\section{Éditeur}

Rails \& histoire

\section{Édition imprimée}

Date de publication : 1 février 2003

Pagination : 43-67

ISBN : 0996-9403

ISSN : 0996-9403

\section{Référence électronique}

Clive Lamming, «Écartement et gabarit : la lente et difficile émergence de normes fondamentales », Revue d'histoire des chemins de fer [En ligne], 26 | 2003, mis en ligne le 19 janvier 2015, consulté le 22 avril 2022. URL : http://journals.openedition.org/rhcf/1939 ; DOI : https://doi.org/10.4000/rhcf.1939 


\section{Écartement et gabarit : la lente et diffi- cile émergence de normes fondamentales}

Le principe même de la circulation de véhicules guidés sur une voie ferrée est très ancien et bien antérieur au « chemin de fer » tel que nous l'entendons aujourd'hui et tel que nous le faisons naitre au début du XIX siècle. L'importance des commencements n'est plus à démontrer en histoire et, plus particulièrement, en histoire des techniques : les commencements du chemin de fer se perdent dans la nuit des temps de l'antiquité, mais l'existence d'un écartement précis n'est qu'une étape très récente qui apparaît au XIX ${ }^{e}$ siècle pour créer le chemin de fer dont la Révolution industrielle a besoin. La lente et difficile naissance de la normalisation de l'écartement des voies et des gabarits est un exemple intéressant en histoire des techniques, car elle montre comment naissent et progressent les techniques et comment aussi la normalisation, quand elle devient nécessaire économiquement, parvient à faire un difficile choix, quitte à éliminer arbitrairement (et injustement) des pans entiers d'une technologie sans autre raison que celle de la compatibilité ou de l'interopérabilité.

\section{Les effets désastreux des "guerres des normes"}

L'histoire des techniques nous apprend que toute technique développée et répandue connaît, assez rapidement, un stade crucial qui est la guerre des normes : nous avons tous présentes à l'esprit la guerre des formats dans le domaine de la photographie qui voit le triomphe du 24 x 36 à la fin des années 1930, la guerre des normes dans le domaine de la télévision avec les systèmes PAL et SECAM européens ou le fameux NTSC américain (dont les réglages si délicats lui valurent le surnom de «Never The Same Color» de la part des techniciens de l'époque !), ou encore les très récentes batailles commerciales et techniques dans le domaine de l'informatique entre la technologie Mac et celle des $P C$. L'automobile en a connu de semblables avec les dimensions des roues et des pneumatiques ou les normes des équipements techniques comme l'allumage. Plus anciennement, l'histoire des armes, elle aussi, est parcourue par le problème de la normalisation des calibres. L'histoire très riche de l'électricité n'a pas manqué de poser les problèmes issus des choix fondamentaux des types de courants (continu, alternatif), des tensions et des fréquences et, jusqu'à aujourd'hui encore, le problème des normes des prises de courant reste d'actualité pour ceux qui veulent voyager avec leur rasoir électrique... 
Ces guerres des normes ont, à nos yeux, quatre caractéristiques désastreuses :

- Elles surviennent trop tard pour être pleinement efficaces.

- Elles n'opèrent que par amputation.

- Elles coûtent très cher à l'ensemble des acteurs ou des entreprises.

- Elles ne font pas toujours triompher la meilleure des technologies mais seulement celle pratiquée par le groupe industriel le plus puissant ou sachant le mieux « communiquer».

\section{Le chemin de fer n'a pas été épargné}

Même si le chemin de fer n'a pas connu, réellement, de guerres internes de normes comme il y a en a eu dans d'autres domaines plus récents avec une volonté de monopole et d'élimination de systèmes considérés comme adverses, il n'a pas échappé à des choix cruciaux laissant pour solde de tout compte ces quatre effets. La présence de nombreux écartements et gabarits en est un exemple qui a perduré pendant toute l'histoire des chemins de fer pour n'avoir pas été pris en compte pendant la longue et lente gestation du système ferroviaire dans la nuit des temps.

L'écartement n'est nullement l'objet d'une lutte pour devenir le seul écartement mondial : en 1925, comme nous pouvons le constater dans un tableau paru dans la Revue générale des chemins de fer, il existe environ 37 écartements dans le monde faisant l'objet d'une pratique effective. L'écartement normal domine, certes, si l'on se réfère au total du kilométrage des voies l'utilisant (notamment grâce à l'impact de l'immense réseau des États-Unis), mais on ne peut pas dire qu'il a dominé grâce aux effets d'une "guerre », c'est-à-dire d'une lutte avec pour objectif de détruire l'adversaire. Chaque ligne, puis chaque réseau, puis chaque pays a, initialement, suivi ses propres choix dans la mesure où, jusque vers 1880 du moins, on n'envisageait nullement un trafic international ou à l'échelle d'un continent entier.

Lorsque Cecil Rhodes, au début du XIX ${ }^{e}$ siècle, veut unir Le Cap au Caire par le rail et créer une grande ligne qui va développer l'Afrique, il se heurte au problème de la multiplicité des écartements africains et ne songe nullement à transformer les voies des réseaux impliqués dans le projet, mais à les utiliser telles quelles. La péninsule ibérique n’a pas, jusqu'à présent, envisagé une telle opération pour son intégration dans l'Europe et reste fidèle aux techniques du changement des essieux tout comme le réseau russe. 
Mais il y a bien quelques brillantes exceptions à la règle avec, d'une part, ce que j'appellerai des standardisations menées par la force publique (cas des Etats américains du Sud après la guerre de Sécession, par exemple) et, d'autre part, avec de véritables guerres menées par des réseaux voulant imposer leur propre norme (cas célèbre de la guerre des écartements menée par le Great Western Railway en Angleterre, sur lequel nous reviendrons). La première manifestation d'un souci de normalisation est la conférence de Berne ouverte le 10 mai 1886 sur laquelle nous reviendrons également.

\section{L'explication: I'ancienneté du principe technique du chemin de fer}

Les Carthaginois ont utilisé des systèmes de plans de roulement en bois pour le déplacement et la mise en place de leurs machines de guerre. Une poutre posée horizontalement et offrant un chemin de roulement uni est donc l'ancêtre du rail utilisé dans le chemin de fer : il n'y a donc rien d'étonnant à ce que l'on trouve des traces de cette pratique dans tous les cas où il fallait mouvoir des charges lourdes, jusque dans l'antiquité. Les Romains ont utilisé le principe du véhicule guidé sur leur célèbre système de voies partout où la sécurité l'exigeait : des ornières étaient taillées dans la chaussée empierrée pour le guidage des roues des chariots sur les routes bordant un précipice, comme sur la route du Pirée à l'Agora d'Athènes ou dans les environs de Syracuse en Sicile. On pense que le transport des bateaux à travers l'isthme de Corinthe se faisait sur un système analogue de glissement sur bois. Le Moyen Âge et la Renaissance ne perdent pas l'usage du système à ornières, mais le font évoluer sous la forme de rails en bois. La Cosmographie universelle de Sébastien Munster, dont la première édition est parue à Bâle en 1550, décrit le système de wagonnets sur rails de bois utilisé dans les mines de Leberthal. A l'époque on trouve des systèmes analogues en Transylvanie, dans le Tyrol. L'ouvrage De re metallica d'Agricola parait en 1556 et décrit un système de wagonnets circulant sur des rails en bois et guidés par des ergots. Très répandu dans les mines d'Allemagne ou des Flandres, ce système a peut-être permis la naissance de la roue à boudin de guidage par usure naturelle des roues en bois sur les rails, surtout quand ces derniers étaient garnis de bandes de fer pour prolonger leur durée. Dans l'ensemble de ces cas, il n'est pas question de normalisation générale : les plans inclinés des Égyptiens sont construits au coup par coup et à la demande sur les chantiers, les voies minières du Moyen Âge sont des constructions artisanales faites directement par les intéressés en fonction des caractéristiques de la mine. Toutefois on 
signale couramment que les dés de pierre des passages pour piétons dans les villes romaines imposent bien une norme obligatoire et réglementée, imposée aux constructeurs de chariots pour une ville donnée.

En 1602, l'exploitant de mines anglais Huntingdon Beaumont aurait, d'après plusieurs documents de l'époque, installé un système de rails desservant les mines des environs de Newcastle et capable de transporter le charbon jusqu'aux ports des rivières ou de la mer. Les wagons, chargés, descendaient vers les ports par simple gravité, avec un homme serre-frein assis à l'arrière, et des chevaux remontaient les wagons vides. Une mine possédait, en 1690, un parc de plus de 600 wagons : le système fut donc très développé et dura longtemps. L'écartement de ses rails n'est pas connu.

\section{La naissance d'une technique ferroviaire, toujours sans normalisation de l'écartement}

L'ouvrage Les Voyages métalliques ${ }^{1}$ décrit avec précision le système ferroviaire des mines britanniques du XviII ${ }^{e}$ siècle. Le charbon est transporté dans des wagons de grandes dimensions, d'une capacité de plusieurs tonnes, roulant sur des voies avec des rails en bois. Les roues ont un boudin de guidage. Des plaques tournantes assurent le changement de direction pour passer d'une galerie à une autre. L'écartement est de 4 pieds, soit $1240 \mathrm{~mm}$. C'est sans doute la première manifestation écrite du souci de donner la valeur d'un écartement, mais nous ne savons pas si ce même écartement a été utilisé ailleurs. On notera toutefois l'utilisation de « valeurs rondes » en pieds, et que l'on retrouvera ultérieurement dans d'autres écartements et dans d'autres pays : 2 pieds, 3 pieds, 5 pieds, 6 ou même 7 pieds. Mais il n'est pas impossible que ces valeurs soient de simples estimations au coup d'œil données par un voyageur.

Le Cours de physique expérimentale de Désaguliers ${ }^{2}$ décrit des wagons pour le transport de grosses pierres entre la carrière de Bath jusqu'à la rivière Avon pour embarquement sur des bateaux. Conçus par Ralph Allen, ces wagons ont un véritable châssis de facture très moderne, d'une longueur de 4,60 m environ. Leurs roues sont en fer et ont un boudin de guidage : c'est l'apparition du système de roulement qui caractérisera les chemins de fer du monde entier ultérieurement. Ces roues sont freinées par levier et sabot. Une des roues est montée folle sur l'essieu, et l'autre est solidaire de l'essieu par montage à force sur un

1- Gabriel Jars, Les Voyages métalliques, Paris, 1765.

2- Paru à Londres en 1734. 
carré : ce système facilite l'inscription et le parcours en courbe à faible rayon. Le système de freinage comprend aussi un verrou de blocage pour l'immobilisation permanente des wagons.

Le premier véritable réseau ferroviaire est établi autour des mines de Coalbrookdale par Abraham Darby entre 1750 et 1763, une œuvre prolongée par son gendre Richard Reynolds jusqu'en 1771 avec, cette fois, l'utilisation exclusive de rails en fonte de $1,80 \mathrm{~m}$, dont 800 tonnes furent posées. La longueur du réseau dépassa $30 \mathrm{~km}$.

John Curr invente, vers 1796, le premier «tram road », c'est-àdire un système de voie ferrée avec des rails comportant un rebord de guidage pour des wagons munis de roues à table de roulement plat sans boudin. Le système est très développé, avec des changements de voie et des évitements. Les rails en bois, puis en fonte pour des raisons de résistance à l'usure, sont posés sur des traverses en bois, puis sur un système de dés en pierre. Les rails du système Curr sont appelés " platerails ». C'est le fondeur James Outram qui les fournit. On pense que le mot « tram road », désignant couramment le système Curr, et donnant notre moderne tramway, serait issu du nom du fondeur, "Outramroad» voulant dire « route Outram» en langue anglaise $e^{3}$. Mais il est intéressant de noter que l'utilisation de rails plats, larges, et dotés d'un rebord de guidage, permet la circulation de charrettes classiques (donc, de percevoir des péages!), avec une large tolérance en matière d'écartements. Des rails plats et très larges permettent, en effet, de recevoir le plus grand nombre de charrettes possible et les rebords de guidage ne sont là que pour remettre dans le droit chemin les roues d'une charrette qui aurait trop dévié. Acceptant toutes sortes de véhicules routiers, la voie n'a pas, à proprement parler, un écartement donné : c'est une piste routière dotée de rebords de guidage.

En 1789, l'anglais Jessop invente le rail moderne ou « edge rail» entièrement métallique avec roue à boudin de guidage. Marc Seguin, dans la partie historique de son traité de $1839^{4}$, attribue à un monsieur Sessop cette invention dont il confirme la date de 1789 (sans doute estce bien Jessop) et précise que l'inventeur « a transporté le rebord (de guidage) sur les roues » car « la poussière et la boue, s’accumulant dans l'angle que formait ce rebord, nuisaient à la circulation ». Mais Jessop

3- Interprétation contestée par Gustavo Reder dans Le Monde des locomotives à vapeur, Paris, Éditions Office du livre, 1974, p. 11, qui estime que le mot vient du latin trames (chemin, sentier) déjà utilisé dans ce sens au Xvi ${ }^{\circledR}$ siècle.

4- Marc Seguin, De l'influence des chemins de fer et de l'art les tracer et de les construire, Lyon, Éd. Pitrat, 1839, réimprimé en 1887. 
fait-il autre chose que réinventer ce qui a existé déjà dans les mines allemandes et, surtout, sur le chemin de fer de Bath conçu en 1734 par Ralph Allen?

En France l'usage des rails se répand dans quelques grands centres industriels de la fin du XviII siècle comme Le Creusot, Indret, Anzin, les mines de plomb de Pallouen. Il s'agit en général de rails de bois garnis de plaques de fonte sur lesquels circulent des trains de wagons à bascule tirés par des chevaux, avec roues à boudin de guidage. Ces courtes lignes ne sont que des « lignes affluents » des rivières ou des canaux, construits à des écartements très divers. On les appelle souvent des « canaux secs ».

En 1791 le Français Faujas de Saint-Fond écrit son Voyage en Angleterre, Écosse et aux Iles Hébrides et décrit le système de transport du charbon entre les mines et le port de Newcastle, doté de voies se prolongeant sur une estacade surélevée sous laquelle se placent les navires ${ }^{5}$. Le charbon est déversé directement dans une trémie qui remplit instantanément la cale du navire. D'après cet auteur, à cette époque, le réseau ferré britannique dépasserait $600 \mathrm{~km}$ de lignes, dont la moitié sous terre dans les mines galloises ou anglaises, mais dont l'ensemble ne semble répondre à aucune norme générale en matière d'écartement et de roulement sinon que d'être dans un écartement d'environ 4 pieds.

Trevithick (1771-1833) et Vivian mettent au point une voiture à vapeur dont la forme est inspirée de celle des diligences. Le moteur manque de puissance et ne pouvait vaincre la résistance au roulement des roues sur les routes. En mars 1802 les deux inventeurs prennent le brevet de l'emploi de voitures à vapeur roulant sur des « chemins de fer à rails ", un pis-aller pour eux, non essayé concrètement. Leur défaut de persévérance leur a fait manquer la fortune : le chemin de fer à traction à vapeur dont ils avaient, en fait, déposé le brevet d'invention! Richard Trevithick est bien l'inventeur de la première locomotive à vapeur sur rails, construite en 1803 et essayée à Merthyr Tydvil en 1804. Elle est performante grâce à un moteur à haute pression, léger et puissant, remorquant un train chargé de 10 tonnes de fer et de 70 hommes montés sur les wagons, couvrant $14 \mathrm{~km}$ en $45 \mathrm{mn}$. L'association voie ferrée et locomotive est enfin concrètement créée : c'est le démarrage du chemin de fer moderne. Mais nous sommes toujours dans le système à rails plats avec rebord de guidage (intérieur, cette fois) et roues ordinaires. Les normes de roulement, notamment en matière d'écartement, ne

5- S'agit-il du système Huntingdon Beaumont de 1602, toujours en service deux siècles plus tard? Si oui, ce serait un record de longévité ferroviaire non battu à ce jour! 
sont pas cruciales et il semble que, pour ces années 1804-1805, le choix entre le système à roues ordinaires sur rail plat à boudin de guidage, d'une part, et, d'autre part, la roue à boudin de guidage sur rail à champignon n'ait pas encore été fait définitivement. La voie à rails plats semble une commodité pour l'essai de véhicules à vapeur parce qu'offrant une résistance au roulement moindre qu'une route : il ne semble pas encore acquis que la traction à vapeur se fera exclusivement sur des voies ferrées (fig. 1).

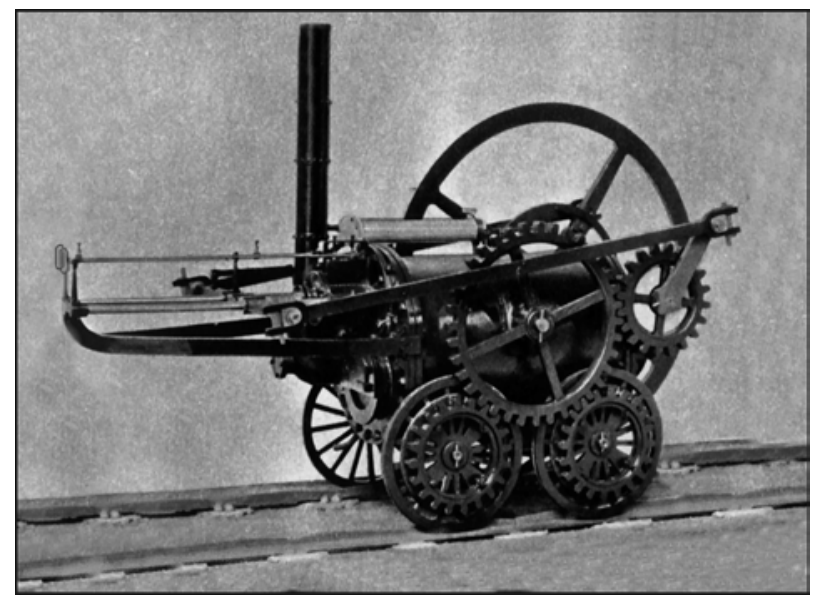

Figure 1. La locomotive Trevithick en 1804. La voie est faite de rails courts, en fonte moulée, posée sur des dés en pierre. Noter les roues plates et le guidage par rebord interne sur le rail. En 1805 Trevithick choisit définitivement le rail plein et la roue à boudin de guidage. $\mathrm{Cl}$. The Locomotive Magazine, coll. Cl. Lamming

En 1805 Trevithick construit une $2^{\mathrm{e}}$ locomotive à Newcastle, destinée à la mine de Wylam. Munie d'un unique cylindre placé dans la chaudière, à l'arrière, cette locomotive doit être accompagnée... à pied par son conducteur qui marche à côté d'elle! Essayée, elle est un échec et est transformée en machine fixe. Mais les dessins d'époque montrent bien que cette locomotive, contrairement à la précédente, utilise le système actuel de roues à boudin de guidage intérieur et de rails à champignon. Le basculement technique a-t-il été fait à ce moment?

Enfin en 1808 Trevithick construit une locomotive très simple, la Catch me who can ("M'attrape qui peut »), pesant 7 tonnes, à 4 roues et un cylindre vertical arrière, et échappement par la cheminée. Elle est utilisée 
dans un manège public payant à Londres, roulant à $20 \mathrm{~km} / \mathrm{h}$ sur une voie circulaire à rails à champignon. Elle marque la fin des essais de Trevithick qui est découragé et ruiné. Trevithick a mis en évidence le problème de la fragilité des rails qui se brisent et du manque d'adhérence de la locomotive qui patine.

Blenkinsop met au point, en 1811, une locomotive à roue dentée s'engrenant sur une crémaillère moulée avec les rails. Utilisée dans la houillère de Middleton, la locomotive est performante mais la crémaillère s'use rapidement sous l'effort de traction et les frottements des roues dentées. Toutefois Blenkinshop utilise aussi la roue à boudin de guidage intérieur et le rail à champignon.

\section{Mais pourquoi un écartement de 4 pieds $81 \frac{1}{2}$ pouces ?}

En 1814 entre en scène un homme dont l'action persévérante sera déterminante dans l'histoire des chemins de fer : George Stephenson (1781-1848). Modeste mécanicien, n'ayant aucune formation d'ingénieur comme Trevithick ou Hedley, il se fait remarquer par ses talents concrets et son habileté mécanique. Il parvient à intéresser Lord Ravensworth, propriétaire des mines de Killingworth, au financement de ses recherches et essais. Nicholas Wood, ingénieur des mines, remarque Stephenson et le présente aux propriétaires des houillères de Darlington pour qui Stephenson construit la locomotive Blücher à transmission par engrenages placés sous la chaudière et circulant sur des rails à champignon. Les engrenages, fragiles, posent des problèmes et Stephenson comprend qu'il faut bannir à jamais tout engrenage dans une locomotive, quitte à multiplier les cylindres et les roues motrices. Stephenson construit en 1815 la locomotive connue sous le nom de Killingworth Locomotive dont le fonctionnement est satisfaisant. Elle est sur deux essieux, reliés par une transmission à chaînes. Chaque essieu est entrainé par son propre cylindre à double effet placé sur le dessus de la chaudière et actionnant de longues bielles verticales. L'écartement du réseau de Killingworth est de 4 pieds 8 pouces (1 $422 \mathrm{~mm}$ ) qui, d'après l'historien anglais George Freeman Allen ${ }^{6}$, est commun à beaucoup de réseaux houillers du nordest anglais depuis le XVIII siècle. Peu connue, elle est pourtant dotée d'un système de transmission caractéristique et déterminant celui de l'ensemble des locomotives à venir : tout est effectué par bielles, qu'il s'agisse de l'action des cylindres sur les roues ou du couplage entre les roues. Les engrenages et chaînes ont définitivement terminé leur carrière ferroviaire.

6- Freeman Allen, George, Le Grand Atlas des trains, Paris, Atlas, 1995. 
La très célèbre Locomotion est la première locomotive du monde à être engagée sur une ligne publique, la Stockton \& Darlington Railway, en 1825. Chef-d'œuvre de Stephenson, elle accomplit son voyage inaugural devant une foule enthousiasmée qui prend d'assaut le train de charbon et voyage, en habits de cérémonie, sur les wagons. Stephenson a repris l'écartement Killingworth, mais l'aurait augmenté d'un demipouce $(13 \mathrm{~mm})$ pour donner du jeu aux boudins de guidage des roues, ce qui donne le fameux écartement de 4 pieds 8 1/2 pouces.

La Fusée de Stephenson, gagnante du concours de Rainhill en 1829 avec une vitesse de $50 \mathrm{~km} / \mathrm{h}$, conserve cet écartement et marque la fin de l'époque des débuts et l'avènement de la locomotive moderne dont elle a toutes les caractéristiques : chaudière tubulaire (due au Français Marc Seguin), deux cylindres à double effet, attaque directe par bielles, échappement par la cheminée, foyer et plate-forme de conduite à l'arrière, tender séparé remorqué. Mais, surtout, Stephenson saura mettre au point une véritable fabrication industrielle de la locomotive et fournir l'ensemble des réseaux européens avec de nombreux types incessamment perfectionnés.

Il est intéressant de noter que le premier règlement du concours de Rainhill ne fait aucune mention d'un écartement et que l'écartement imposé n'est mentionné que dans un additif publié ultérieurement : le concours se tient sur une portion de la ligne de Liverpool à Manchester, alors en cours de construction sous la direction de Stephenson, et c'est ce qui impose l'écartement de 4 pieds $8 \frac{1}{2}$ pouces à tous les candidats... dont Stephenson lui-même qui se trouvait à la fois juge et partie ! C'est comme fournisseur des réseaux anglais et européens que George Stephenson impose, avec ses locomotives, l'écartement de 4 pieds $81 / 2$ pouces dont on dit qu'il l'aurait choisi un peu au hasard en allant mesurer celui des roues d'une charrette dans une grange voisine de son atelier.

Enfin, et surtout, la roue à boudin de guidage intérieur et le rail à champignon lisse forment le système de référence qui est spécifique de la voie ferrée moderne et la distingue de la route améliorée par des rails plats. Choisi par Stephenson pour la ligne de Stockton à Darlington, puis pour celle de Liverpool à Manchester, ce système reste définitivement acquis au le chemin de fer. Mais il pose, désormais, le problème de l'écartement qui doit être précis à quelques millimètres près, et qui doit être commun à toutes les lignes désirant s'intégrer dans un réseau.

Notons, pour terminer, une aventure intéressante ${ }^{7}$ qui montre à quel point le problème de l'écartement n'était pas encore perçu par les ingénieurs. En 1816, les ingénieurs allemands Krigar et Eckard achètent,

7- Contée par Gustavo Reder, op. cit., p. 20-21. 
pour le compte des mines et des fonderies royales de Haute-Silésie, une locomotive du système Blenkinshop qui est livrée le 23 octobre de la même année, en pièces détachées. Lors du montage de la locomotive, on découvre que l'écartement des roues est de $914 \mathrm{~mm}$, soit sensiblement 3 pieds anglais, mais les voies des mines et des fonderies sont, elles, à l'écartement de $1294 \mathrm{~mm}$ : la différence est de $380 \mathrm{~mm}$, mais ne semblait pas avoir été l'objet d'une préoccupation. De toutes manières la machine, après mise à l'écartement souhaité, ne fonctionna pas et termina sa vie comme pompe à vapeur fixe. L'écartement, pas plus qu'aucune notion de norme, n'était pas encore " entré dans les esprits » et il semble que cet incident fut loin d'être un cas isolé à l'époque.

\section{La première apparition publique du problème de l'écartement}

Le problème de la normalisation de l'écartement n'est pas, à vrai dire, directement posé par des exploitants des lignes qui, pour le moment, ne songent nullement à se relier les uns aux autres pour former un réseau national cohérent. Même Marc Seguin, dans son De l'influence des chemins de fer ${ }^{8}$, ne mentionne aucun problème de choix d'écartement et considère comme acquis l'écartement de Stephenson qu'il qualifie de « voie de 1,50 mètre » (p. 230), appellation que l'on retrouve aussi dans le traité de G. Humbert à la fin du siècle?. En 1842, l'ensemble des lignes anglaises est entre les mains de 50 compagnies indépendantes et privées qui n'ont pratiquement aucun lien technique ou administratif entre elles et qui, surtout, ne sont l'objet d'aucune tutelle de la part de l'État. En 1852 le nombre est passé à 75 compagnies. Ce n'est que vers 1865 qu'une majorité d'entre elles font partie du Railway Clearing House qui est un organisme administratif chargé de gérer les charges et les bénéfices provenant des premiers courants de trafic entre différents réseaux : l'usage de voyages ou d'expéditions sur l'ensemble du réseau ferré britannique commence à entrer dans la vie courante et à imposer la circulation de voitures et de wagons d'un réseau à un autre. Le problème est d'abord posé sur le plan financier il n'est pas encore question de normes techniques autres que celles permettant la circulation du matériel roulant remorqué. L'interpénétration des réseaux par les locomotives n'est pas encore admis, chaque réseau conservant sur son territoire ses locomotives et ses équipes de conduite, sa signalisation, ses pratiques professionnelles, jalousement gardées pour soi.

\section{8- Seguin, op. cit.}

9- La valeur de 1,50 m peut être obtenue en mesurant l'écartement entre les axes des rails si les champignons ont une largeur de $65 \mathrm{~mm}$. 
Toutefois, en 1845, le gouvernement britannique est contraint de se mêler du problème des écartements : dans un pays où l'on compte 13 écartements, le 4 pieds $8 \frac{1}{2}$ pouces domine du fait de l'influence de Stephenson qui a fourni des locomotives à une majorité de réseaux (et qui oblige par la force des choses les futurs fournisseurs de ces mêmes réseaux à adopter le même écartement) tandis que le fameux 7 pieds préconisé par l'ingénieur Brunel arrive en deuxième position et s'étend sur le sud-ouest de l'Angleterre, le Pays de Galles et remonte jusqu'à Wolverhampton en direction du Nord. En 1846 la voie de 7 pieds totalise environ 275 miles, soit plus de $12 \%$ des 2225 miles du réseau anglais. L'Irlande se voit interdire la construction de lignes autres qu'à l'écartement 5 pieds 3 inches dans lequel elle a commencé son réseau: l'expérience anglaise est mise à profit pour empêcher la multiplication des écartements, mais ce pays en sera bien malgré tout doté de plusieurs.

Sur le continent européen l'expérience anglaise de l'anarchie des écartements ne sert guère de leçon et l'on trouve de nombreux écartements différents. On peut noter que l'Europe de l'époque est encore très enfermée dans ses frontières et ses pratiques nationales et qu'un grand réseau international, s'il est espéré par les esprits les plus ouverts et les plus entreprenants, n'est pas ce qui dicte les choix de chaque pays européen. L'adoption commune de l'écartement de Stephenson est beaucoup plus un fait technique, lié à l'achat de ses locomotives. Si les industriels anglais, dans leur ensemble, Stephenson compris, produiront bien à la demande des locomotives pour les écartements des pays qui ont, pour diverses raisons techniques (notamment de dimensionnement des chaudières pour avoir une plus grande puissance de traction), choisi des écartements plus larges (comme la péninsule ibérique ou la Russie), il est certain qu'à l'époque des démarrages des réseaux continentaux, là où Stephenson a carte blanche il livre des locomotives dans son écartement "maison» : c'est le cas pour la première ligne belge de Bruxelles à Malines à partir de laquelle se constituera le réseau de ce pays, ou encore de la première ligne allemande de Nuremberg à Furth (locomotive Der Adler qui n'est autre qu'une Stephenson Patentee), ou de la première ligne italienne (locomotive Bayard), etc. C'est donc beaucoup plus une norme maison d'un constructeur qui fait l'unité des écartements européens que la volonté politique des pouvoirs publics.

Le cas de l'Amérique du Nord d'avant la guerre de Sécession est intéressant : les compagnies bénéficient d'une indépendance totale «à l'anglaise » et ne manquent pas de pratiquer plusieurs écartements très 
divers (1 829, 1 676, 1 448, 1473 mm, etc.) ce qui contribue à la défaite $\mathrm{du}$ Sud en rendant difficiles les déplacements de troupes. Les historiens des techniques noteront que cette défaite est aussi liée à l'absence de normes dans un autre domaine technique, celui des calibres des armes. Les ingénieurs Wallering et Flanders sauront tirer un enseignement de cette leçon de la nécessité des normes en matière d'armement et l'appliqueront aux machines-outils de l'industrialisation américaine de la fin du siècle, surtout avec la production en masse (Ford T).

Le cas du choix russe est aussi intéressant : nullement dû à une xénophobie ou à une erreur de transcription comme on peut l'entendre dire, cet écartement de 5 pieds ( $1524 \mathrm{~mm}$ ) est bien le choix du conseiller américain George Washington Whistler, officier du génie, qui le propose parce qu'il en a déjà la pratique aux États-Unis. Whistler se heurte aux experts du Tsar qui, eux, proposent un écartement de 6 pieds (1 $829 \mathrm{~mm}$ ) pour des raisons de dimensionnement des locomotives, écartement dans lequel la ligne de Tsarskoié-Tsélo a été réalisée. Whistler l'emportera, prestige américain oblige...

A partir des années 1880 le problème de la différence des écartements entre les réseaux de divers pays voisins commence à être posé par le développement du trafic international. Il faut dire que ce trafic n’avait jamais été prévu lors de la construction des chemins de fer, du moins avec une telle intensité : tout au plus, à l'époque, pensait-on les transports par chemin de fer en termes de lignes d'une ville à une autre ou en termes de réseaux régionaux formés par un ou plusieurs embranchements ou raccordements entre les précédentes. De grandes lignes nationales se sont souvent constituées par la mise bout à bout de lignes régionales existantes et, même aux Etats-Unis, on ne voyait pas pourquoi les lignes de certains états du Sud se raccorderaient entre elles, ou se raccorderaient avec celles des états du Nord. Si le milieu du XIx ${ }^{e}$ siècle voit la mise en place des réseaux nationaux, la fin du XIx siècle marque le passage du trafic ferroviaire à une dimension autre, celle de continents entiers.

D'autres problèmes de normalisation vont alors de poser à côté de celui des écartements : les problèmes de normes de roulement, d'attelages, de systèmes de freinage, de systèmes d'alimentation électrique : voilà ce qui, entre autres, donnera du travail à un bon nombre d'ingénieurs et de commissions durant les premières décennies $d u x^{e}$ siècle et, surtout, entre les deux guerres à l'UIC dont le travail est à saluer. 


\section{Un exemple illustre de la guerre des normes : le cas du Great Western Railway}

Fils de Sir Marc Isambard Brunel (1769-1849), un ingénieur d'ascendance française protestante, Isambard Kingdom nait en 1806 et, après des études menées surtout auprès de son père dont il suit les réalisations comme le tunnel sous la Tamise à Londres, il est nommé ingénieur sur le réseau du Great Western Railway en 1833. Brunel, comme certains ingénieurs de son temps, agit en visionnaire et en innovateur. Il est convaincu - à juste titre - que la révolution industrielle qui nait sous ses yeux créera une demande de transport immense et que l'écartement des chemins de fer comme le gabarit des wagons et voitures est nettement insuffisant, qu'il a été prévu avec des vues trop courtes et trop immédiates. Il parvient à persuader les dirigeants du Great Western Railway de briser l'étroite et contraignante norme dite de la voie « normale » de 4 pieds $8 \frac{1}{2}$ pouces $(1435 \mathrm{~mm})$ pour la remplacer par une voie large de 7 pieds $1 / 4(2140 \mathrm{~mm})^{10}$ qui permettra non seulement la circulation de trains plus grands, mais aussi plus rapides. Le conseil d'administration de la compagnie accepte la proposition lors de sa réunion du 25 août 1836, et les actionnaires n'approuvent le projet que le 9 janvier 1839, soit après trois années de débats et de controverses : il semble qu'un certain nombre d'entre eux ait eu des prémonitions ou des craintes quant aux problèmes que la différence d'écartement par rapport aux réseaux voisins ne manquerait pas de poser. Mais l'argument essentiel de Brunel était qu'un écartement différent de celui d'autres compagnies rivales conserverait au Great Western Railway son monopole en empêchant, justement, les concurrents de faire circuler leurs trains sur le réseau. Un seul participant à la commission, un nommé William Cubbit ${ }^{11}$, recommande de transformer entièrement le réseau du Royaume-Uni en voie de 7 pieds « avant qu'il ne soit trop tard».

En effet, il importe de situer le contexte de ce choix. En Angleterre les compagnies de chemin de fer vivent sous un régime de compétition acharnée entre elles, contrairement au cas de la France, par exemple, où l'État, dès les débuts, fixe les règles du jeu. En 1880, par exemple, la relation entre Londres et les 15 plus grandes villes anglaises (exceptées Bristol, Hull et Newcastle) est effectuée par au moins trois compagnies concurrentes qui rivalisent âprement entre elles sur le plan du confort,

10- Beaucoup d'ouvrages présentent cet écartement sous le nom de « 7 pieds ». Nous suivons cet usage sachant qu'en réalité il s'agissait bien d'un écartement de $7 \frac{1}{4}$ pieds.

11- D’après Gustavo Reder, op. cit., p. 82. 
de l'exactitude ou de la vitesse ${ }^{12}$ : en France aucune ville ne jouira d'un tel privilège, sauf Bordeaux pour un temps avec les réseaux de l'État et du PO. C'est dire si chaque compagnie doit lutter avec toutes ses ressources et, au premier plan, celles offertes par la technique en matière de vitesse.

La première ligne en voie large est ouverte entre Londres et Bristol en 1841 et comprend le fameux Box Tunnel long de $2937 \mathrm{~m}$ et creusé au gabarit large. La ligne est prolongée ensuite jusqu'à Exeter, Plymouth, Penzance (par un pont immense créée par Brunel), puis le réseau se développe sur l'ensemble du sud-ouest britannique, incluant le pays de Galles. Gloucester est le premier point atteint comportant un contact avec une ligne à voie normale, mais il semble que les nécessaires transbordements de marchandises ou changements de train pour les voyageurs n'affectent, en fin de compte, qu'un volume restreint. La supériorité de la voie large, en matière de vitesse, est immédiatement démontrée, même avec des locomotives médiocres fournies au début par des firmes peu expérimentées. Les excellentes locomotives de l'ingénieur Gooch, à partir de 1846, feront la réputation mondiale du Great Western Railway, notamment les locomotives à deux essieux porteurs avant type 211.

En 1844 le Great Western Railway s'étend en direction du Nord et atteint Birmingham, une importante gare gérant un trafic voyageurs et marchandises considérable ; c'est bien là que le problème des ruptures de charge et des transbordements se pose d'une manière si exemplaire qu'une commission gouvernementale est instituée, à la demande des voyageurs, pour étudier la question. En 1846 les travaux de la commission se concrétisent par un acte en date du 28 août qui interdit, sur le sol anglais, la construction de voies d'écartement autre que celui de 4 pieds $8 \frac{1}{2}$ pouces $(1435 \mathrm{~mm})$. L'Irlande, pour sa part, pourra construire des voies, mais dans le seul écartement de 5 pieds 3 pouces $(1600 \mathrm{~mm})$ dans lequel elle a commencé son réseau. La commission dispose aussi que le Great Western Railway devra poser un $3^{\mathrm{e}}$ rail sur ses lignes à voie large construites en direction du Nord, cela de manière à permettre la circulation aussi bien du matériel roulant à voie normale qu'à voie large ${ }^{13}$.

12- Jack Simmons, Rail 150 - The Stockton \& Darlington Railway and What Followed, Londres, Eyre Methuen, 1975.

13- D'après O.S. Nock (Railways then and now, Londres, Paul Elek, 1975), la commission aurait été fortement influencée par les autorités militaires qui craignaient que les transbordements soient un obstacle pour la concentration rapide des troupes en cas d'invasion du Royaume-Uni par l'armée d'un pays continental. La situation instable de l'Europe des années 1840 préoccupait les états-majors anglais. 
En 1867 le réseau à voie large du Great Western Railway se compose de $2300 \mathrm{~km}$ de voies larges, dont certaines à 3 files de rails. C'est l'apogée du système, suivi d'un rapide déclin ${ }^{14}$.

Certes les trains sont beaucoup plus rapides que ceux à voie normale, beaucoup plus stables avec un matériel bien « campé » sur ses roues qui se trouvent pratiquement à l'aplomb du bord des caisses (les caisses n'étant qu'à peine plus larges que celles de la voie normale). Les locomotives aux dimensions plus généreuses sont plus puissantes, plus faciles à entretenir du fait d'un espace plus important entre les longerons du châssis, et la disposition des cylindres entre les longerons, facteur de stabilité, n'impose pas, comme avec les locomotives à voie normale, des cylindres de petit diamètre. En 1846, des locomotives type 111 à un seul essieu moteur avec des roues de $2438 \mathrm{~mm}^{15}$ assurent des moyennes de $100 \mathrm{~km} / \mathrm{h}$ entre Londres et Swindon en tête de trains de 100 tonnes, et de $90 \mathrm{~km} / \mathrm{h}$ entre Londres et Exeter sur $312 \mathrm{~km}$ - des vitesses que les trains anglais d'aujourd'hui envieraient... Ces performances sont, de très loin, supérieures aux meilleures performances en voie normale. Mais les inconvénients posés par les transbordements, par l'impossibilité de créer des trains ou des voitures directs prolongeant leur parcours sur les réseaux voisins ou l'impossibilité de recevoir ces mêmes trains sur le réseau à voie large viennent facilement à bout des avantages présentés par l'écartement de 7 pieds. En 1869 le Great Western Railway se prépare à tourner la page et à reconvertir l'intégralité de son réseau à la voie normale, cela d'autant plus que les locomotives à voie normale ont fait des progrès considérables et que leurs performances ne sont plus, désormais, strictement liées à leurs dimensions. Ce sont surtout les travaux de Thomas Crampton, lui-même issu du réseau du Great Western Railway et élève de Gooch, qui donneront aux locomotives à voie normale l'occasion d'égaler les locomotives à voie large en matière de vitesse (fig. 2).

Dès cette année 1869, c'est chose faite pour la ligne Wolverhampton - Birmingham - Oxford dont il a suffi de déposer le $3^{\mathrm{e}}$ rail extérieur. En 1872 la plupart des lignes du pays de Galles sont traitées, ou dotées d'un $3^{\mathrm{e}}$ rail comme c'est le cas pour la ligne Swindon - Didcot dont le $3^{\mathrm{e}}$ rail permet, désormais, des liaisons directes en voie normale entre Londres et le Pays de Galles par Gloucester. Vingt ans plus tard, le

14- O.S. Nock écrit, pourtant, que le Great Western Railway eut la possibilité de parachever des lignes à voie large jusqu'en 1877, le texte du décret étant volontairement assez flou en ce qui concernait les lignes en cours de construction (ibid.).

15- Certaines locomotives auront des roues de $2750 \mathrm{~mm}$, comme les types 212 de 1873 , et même de 3 mètres. 
20 mai 1892, le dernier train de voyageurs à voie large quitte la gare londonienne de Paddington, le fameux Cornishman de $10 \mathrm{~h} \mathrm{15,} \mathrm{et} \mathrm{le} \mathrm{tout}$ dernier train, toutes catégories confondues, est le train postal de nuit reliant Penzance à Londres arrivant tôt le matin du 21 mai 1892 (fig. 3).

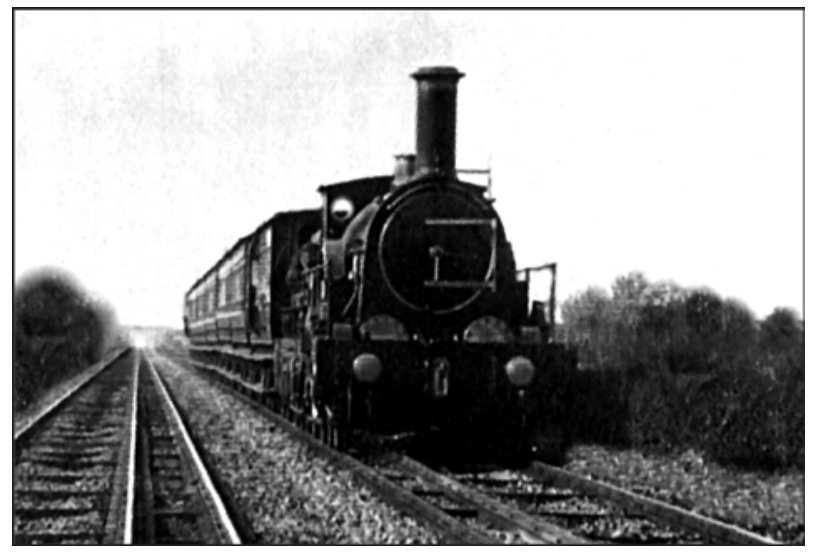

Figure 2. Vue d'un train en voie large de Brunel vers 1890. Le gabarit du matériel n'est guère plus large que l'écartement de la voie et n'offre donc pas plus de possibilité de transport que le matériel à voie normale. Noter le troisième rail préparant la conversion qui sera achevée en 1892. Cl. d.r., coll. Cl. Lamming,

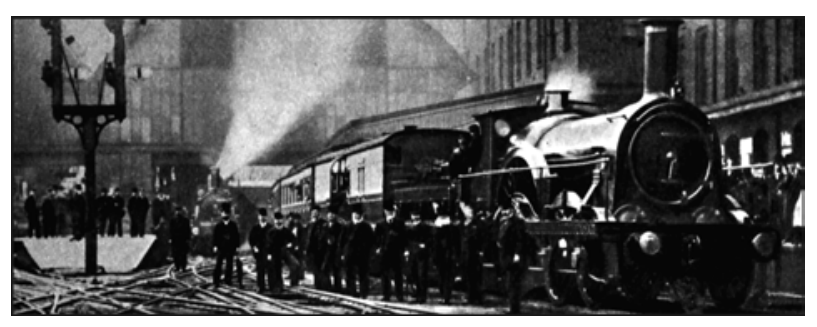

Figure 3. Le dernier train en voie large à Paddington en 1892 : noter la complexité des appareils de voie. Cl. The Locomotive Magazine, coll. Cl. Lamming 
Le coût de l'opération a été considérable et est durement ressenti par un réseau qui, comme beaucoup de réseaux anglais, connait une sombre période durant les années 1860 et 1870 avec beaucoup de déraillements et de catastrophes, de revendications des cheminots concernant leurs conditions de travail, d'attaques de la part des milieux politiques à propos de la politique financière des compagnies, un mécontentement général des usagers à cause de la dégradation des services. Entre 1860 et 1900, les coûts de l'exploitation du réseau anglais ont augmenté de $63 \%{ }^{16}$ du fait de la nécessité de perfectionner le matériel roulant (application du frein continu, des enclenchements, amélioration de la sécurité à bord des trains, aménagement de l'intercirculation entre les voitures, etc.). Toutefois le Great Western Railway se classe, pour ce qui est du revenu net du capital, à la $5^{\mathrm{e}}$ position des 15 grands réseaux anglais, avec un revenu de $5 \%$ pour 1894 (les meilleurs étant à $6 \%$, et les derniers à... $0 \%{ }^{17}$, ce qui prouve que ce réseau a toujours les reins solides et a pu s'offrir le luxe de cette dépense.

Le coût de l'opération est surtout dû au matériel roulant. Les locomotives à voie large forment un parc de 549 exemplaires en 1869, à l'apogée du système, et encore de 67 exemplaires (purement et simplement ferraillés) en 1892. Les voitures sont au nombre de 1590 en 1869 et encore de 126 en 1892. Les wagons sont au nombre de 8651 en 1867, et il faudra purement et simplement ferrailler 2477 wagons en 1892. Le matériel transformable est composé de seulement 125 locomotives (transformation par déplacement des boites d'essieu de l'extérieur vers l'intérieur des longerons), 426 voitures et 792 wagons en $1892^{18}$. Le stockage du matériel roulant demande 18 kilomètres de voies spécialement aménagées à Swindon, où il restera entreposé des années durant. On peut imaginer le coût pour un réseau de taille analogue, comme la région Ouest de la SNCF d'aujourd'hui, par exemple, que représenterait la perte subite de 67 locomotives, 126 voitures et 2477 wagons en une seule année !

Pour ce qui est des opérations sur les voies, les choses sont facilitées par le fait que la presque totalité des lignes à transformer est en double voie, ce qui permet une circulation sur une voie unique temporaire pendant que les travaux affectent l'autre. Toutefois il existe de longues voies uniques comme celle de Exeter à Falmouth, longue de $270 \mathrm{~km}$ : l'opération se fit entre le samedi 21 à 5 heures du matin et le

16- Simmons, op. cit.

17- Revue générale des chemins de fer, mai 1895, p. 260.

18- Revue générale des chemins de fer, septembre 1892, p. 106 et sq. 
dimanche 22 mai 1892 au soir, avec 5000 hommes répartis tout au long de la ligne après le passage du dernier train en voie large, récupérant tout le matériel se trouvant sur la ligne. Des équipes de 50 à 70 hommes doivent, en 31 heures, traiter des sections de 3000 à $4000 \mathrm{~m}$ : elles doivent enlever le ballast, scier les longrines Brunel soutenant les rails, riper les longrines, boulonner des tiges en fer maintenant le nouvel écartement.

Dans les gares et bifurcations, les appareils de voie étaient préparés à l'avance. Le rétrécissement des voies a demandé l'élargissement des quais en îlot. Un certain nombre de ponts métalliques ont dû être modifiés dans la mesure où leurs longerons supportaient directement les rails. Aujourd'hui encore on peut admirer, sur les lignes de l'ancien Great Western Railway entre Londres et le pays de Galles ou les Cornouailles, des ouvrages d'art aux dimensions surprenantes, notamment les entrées de tunnel dont la section est souvent deux fois plus haute que celle des trains actuels. C'est tout ce qui reste de cette aventure remarquable qui permit de dire, à l'époque, que le Great Western Railway était vraiment "great», contrairement au noms des autres compagnies usurpant cet adjectif voulant dire "grand» au sens de " grandiose ».

\section{De la normalisation de l'écartement à celle du gabarit}

L'épisode de la bataille des écartements en Angleterre ne fait que cacher l'inexistence de la deuxième donnée fondamentale : le gabarit. Nous donnons, en annexe, quelques-unes des positions soulevées pendant la tenue de la commission de normalisation (annexe 1). Un certain nombre de participants objecte que la voie large de Brunel n'offre de capacités de transport qu'à peine supérieures sinon comparables à celles de la voie normale. La raison est très évidente si l'on regarde une des gravures d'époque montrant des trains en voie large : en effet les caisses du matériel roulant ne sont guère plus larges que la voie et les roues sont bien soit à l'aplomb des parois des caisses, soit franchement à l'extérieur des caisses, comme l'étaient les roues des diligences et des charrettes de l'époque. Le choix d'une voie large n'est nullement motivé, chez Brunel, par l'augmentation de la capacité de transport (comme ce sera le cas pour les partisans d'un réseau européen en voie de 3 mètres sous le régime nazi) mais seulement par une plus grande facilité de conception des locomotives, leur meilleur dimensionnement, leur plus grande puissance, leur plus grande rapidité.

Sil'on regarde les locomotives anglaises en voie normale de l'époque, on constate qu'elles sont à peine plus larges que la voie et que leur 
mouvement intérieur est entièrement compris entre les roues. Seul le matériel roulant remorqué, notamment les voitures à caisse galbée, se risquent à un léger débordement. Le seul élément intervenant pour donner un peu d'ampleur au gabarit est la hauteur des cheminées qui dépasse souvent $4,50 \mathrm{~m}$.

Nous n'avons pas trouvé de texte de normalisation du gabarit anglais, mais en observant les cotes portées sur des dessins anciens (coupes de voitures), il semble que l'ensemble des compagnies pratique, vers 1870, un gabarit hors tout de 9 pieds. Les voitures sont construites avec une caisse dont la largeur est variable selon les compagnies, entre 7 pieds 5 inches $(=2,22 \mathrm{~m})$ et 8 pieds 4 inches $(=2,50 \mathrm{~m})$, mesures prises au plancher et sans compter un débordement possible pour les caisses galbées. Vers la fin du siècle, une curieuse pratique consistant à scier en longueur les caisses de 7 pieds 5 inches pour les élargir à 8 pieds 4 inches va s'instaurer pour augmenter la capacité des voitures, faisant asseoir les voyageurs par rangées de 6 au lieu de 5 dans les compartiments ${ }^{19}$. On voyage quand même serré, si l'on songe que l'on s'assied à cinq sur une banquette contenue dans une caisse de 2,22 $\mathrm{m}$ de large, épaisseur des cloisons comprises, soit environ une quarantaine de centimètres par personne...

L'explication historique de cette pratique du sciage en long des caisses, à nos yeux, est bien celle de l'absence de toutes normes pour le gabarit. Quand, à partir des années 1860, les circulations directes longue distance de voitures passant d'un réseau à un autre deviennent habituelles, les réseaux construisent des voitures en respectant le gabarit du réseau le plus restreint puis, quand le réseau le plus restreint progresse techniquement et augmente son gabarit, les autres réseaux peuvent alors engager des voitures plus larges : il ne leur reste plus, éventuellement, qu'à élargir leurs voitures plus anciennes (fig. 4).

Ce n'est que le 10 mai 1886 que se réunit à Berne une conférence internationale destinée à "arrêter les bases d'une unité technique des voies et du matériel $»^{20}$ où sont représentés les réseaux de la France, de l'Allemagne, de l'Italie, d'Autriche-Hongrie et de la Suisse, et pas du Royaume-Uni, notons-le. La largeur des voies entre les bords intérieurs des rails est fixée à $1435 \mathrm{~mm}$ avec un surécartement en courbe pouvant atteindre $1465 \mathrm{~mm}$. En France ces dispositions sont sanctionnées par un arrête du ministre des Travaux publics en date du 31 mars 1887. Jusquelà les réseaux français pratiquaient un écartement de $1450 \mathrm{~mm}$ en 19- D'après The Locomotive Magazine, volume de 1902, p. 70.

20- G. Humbert, Traité complet des chemins de fer, Paris, Librairie polytechnique, 1891, p. 247. 
alignement - sauf le Midi qui comptait $1445 \mathrm{~mm}$. Les faces internes des roues, en revanche, ont des cotes qui varient d'un réseau français à l'autre.

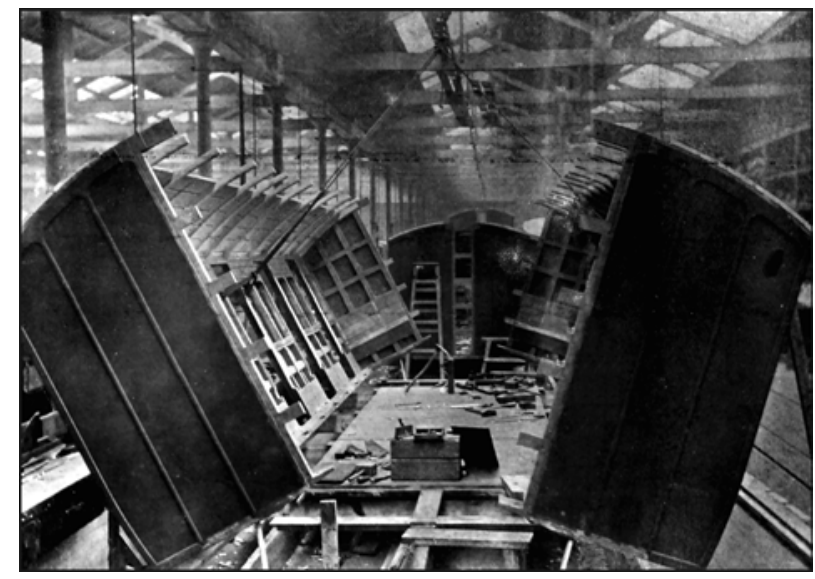

Figure 4. Le "sciage en long" de voitures anglaises, en 1902, dans les ateliers du Great Eastern Railway, sans doute un des documents les plus «surréalistes» de l'histoire technique des chemins de fer: le gabarit de la voiture passera de 7 pieds 7 inches à 8 pieds 6 inches, soit un gain d'un pied $(30 \mathrm{~cm})$, permettant d'accroitre la capacité d'un train de 636 à 768 voyageurs. Cl. The Locomotive Magazine, coll. Cl. Lamming,

Cette conférence, dont nous avons consulté les promulgations, ne se préoccupe pas des gabarits extérieurs du matériel roulant et statue principalement sur les organes de roulement, de choc et de traction. En France, à l'époque, les réseaux ont des gabarits différents se situant entre 2,70 et 2,76 $\mathrm{m}$ pour la largeur des caisses, si l'on se réfère à des plans d'époque du matériel roulant de différentes compagnies. Ces largeurs passeront à 2,90 puis environ $3 \mathrm{~m}$ vers la fin du siècle, chaque réseau ayant ses propres pratiques et, même, construisant des voitures ou des wagons dans des largeurs variables d'une série à une autre.

\section{En conclusion : la difficile normalisation dans le domaine des chemins de fer}

L'ancienneté même des techniques ferroviaires rend d'autant plus difficile tout acte de normalisation. Chaque innovation dans le domaine ferroviaire doit s'insérer dans un ensemble technique ancien, complexe, géographiquement très étendu, faisant appel à une infinité de techniques différentes qui se retrouvent croisées et modifiées au contact les unes des autres. Et chaque innovation met en jeu des composantes 
anciennes avec lesquelles elle doit jouer avant, à son tour, de devenir partie prenante d'un ensemble qui l'engagera pour une durée très longue dans l'avenir.

L'histoire des techniques montre que plus un ensemble technique est ancien, plus son évolution est lente et plus tout acte de normalisation ou changement fondamental est difficile. L'informatique, née il y a peu de temps, connait des bouleversements répétés, une avalanche permanente de nouveautés qui remettent en cause l'ensemble du système jusque dans ses normes fondamentales. L'automobile, née il y a un siècle, ne connait plus de grands changements fondamentaux et le moteur à cylindre et piston est devenu immuable dans son principe.

Le TGV d'aujourd'hui, image même de la modernité et des hautes performances, roule sur un écartement de 4 pieds $8 \frac{1}{2}$ pouces et, même, emprunte entre Lyon et Saint-Etienne le tracé de la voie établi par Marc Seguin pendant les années 1820. Le TGV est sans nul doute l'objet technique le plus performant et le plus innovateur qui bat un record autre que celui de la vitesse : c'est celui de l'ancienneté de certaines de ses normes (fig. 5).

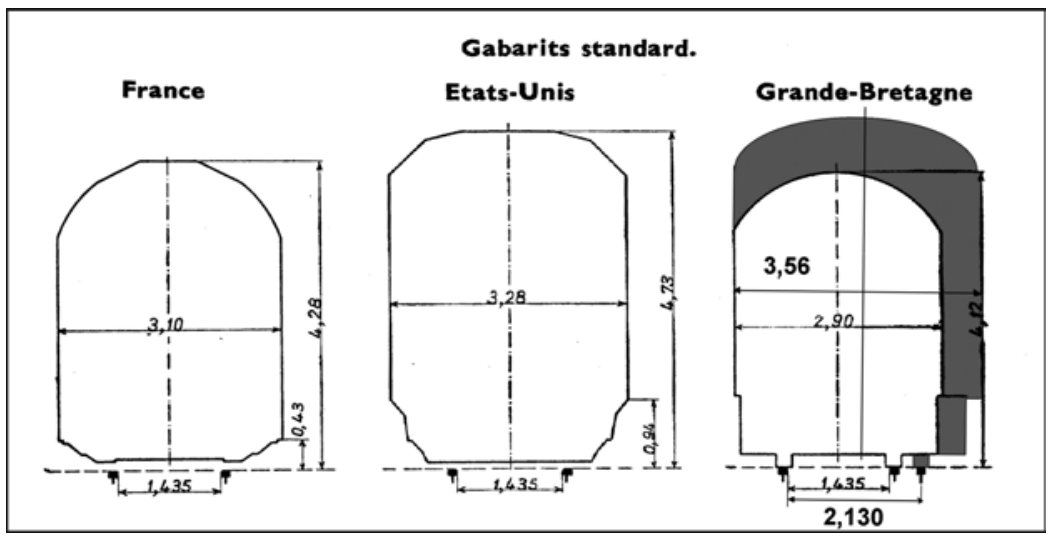

Figure 5. La comparaison entre les gabarits standard actuels américains, français et anglais. Sur le gabarit anglais nous avons projeté le gabarit en voie large de Brunel qui ne donnait qu'une capacité de transport guère supérieure au gabarit voie normale américain, par exemple. Brunel n'avait pas joué la carte du gabarit, mais seulement celle de l'écartement pour établir des locomotives aux cylindres (intérieurs) de plus grand diamètre. C'est sans doute cette faible différence en matière de gabarit qui lui a fait perdre la " battle of gauges " (bataille des écartements) de 1845 : la voie large n'offrait guère mieux, en matière de transport, pour des dépenses infiniment plus lourdes en matière d'établissement et d'entretien. Dessin Cl. Lamming 


\section{Annexe \\ Les débats de la commission de 1845. Quelques prises de position intéressantes pour ou contre la voie de 7 pieds}

Nous avons pu retrouver, dans le tome 8 de 1903 de la revue anglaise The Locomotive Magazine, un compte rendu très complet des débats qui ont eu lieu à la commission de 1845-1846. Plus de soixante années plus tard, on parle encore de la bataille des écartements («The battle of gauges »). Voici quelques positions marquantes.

- John Braitwaithe, ingénieur en chef du Eastern Counties Railway, fait remarquer que son réseau a été construit initialement à l'écartement de 5 pieds et regrette l'adoption ultérieure de l'écartement de 4 pieds $8 \frac{1}{2}$ pouces, car celui-ci donne des locomotives plus petites et moins puissantes et, surtout, d'un accès moins facile pour les opérations d'entretien. Mais il admet que les progrès récents effectués en matière de simplification des locomotives ont rendu l'entretien des locomotives en voie normale aussi aisé, désormais, que l'était celui des locomotives en voie de 5 pieds.

- G.P. Bidder, ingénieur sur plusieurs réseaux dont le Norfolk, pense que des voitures plus larges, comme celles du Great Western Railway, offrent moins de confort, les voyageurs préférant être moins nombreux côte à côte sur les banquettes, et voulant surtout être près des fenêtres : des voitures étroites offrent plus de places près des fenêtres. Des trains plus fréquents sont préférables à des trains plus rares et plus rapides comme ceux de la voie large. Il ne partage pas l'opinion de Gooch du Great Western Railway selon laquelle le coût de la puissance est deux fois moins élevé en voie large qu'en voie normale, parce que, désormais, on sait faire des locomotives en voie normale fournissant autant de vapeur que celles en voie large.

- W. Thernthough, directeur de la traction («locomotive superintendent») de l'Eastern \& Chatham préfère les locomotives à mouvement extérieur et il est partisan d'un écartement d'au moins 5 pieds pour atteindre la vitesse en service de $70 m p h$ (soit $112 \mathrm{~km} / \mathrm{h}$ ).

- J. Hawkshaw, ingénieur sur le Manchester \& Bolton, pense que la largeur de l'écartement n'est nullement inductrice d'une augmentation de la vitesse et que, de toutes manières, on ne pourra 
pas construire à l'avenir des locomotives plus grandes que celles construites actuellement (sic). Le coût de la traction est de 10 pence par mile sur le Great Western Railway et de seulement $63 / 4$ pence sur son réseau en voie normale.

- Joseph Locke aborde la question des voies mixtes à 3 files de rails, qu'il trouve dangereuses, et préconise la construction de voies à 4 files de rails pour que le matériel roulant soit bien centré dans les deux écartements ${ }^{21}$. Il pense que, pour le Great Western Railway, cela coûtera moins cher de tout changer que d'établir des voies à deux écartements, et il rejette le projet proposé par le Great Western Railway d'avoir des caisses de voitures pouvant, à volonté, être posées sur des châssis à voie large ou voie normale, se référant aux dangers occasionnés par le transport des caisses de diligences en France, où ces caisses quittaient les wagons au moindre choc. Il partage aussi la position de G.P. Bidder sur le choix du public en faveur des places près des fenêtres. Enfin il ajoute que les locomotives à roues motrices de 10 pieds (soit 3 mètres de diamètre) du Great Western Railway avaient beaucoup de mal à démarrer et à freiner du fait de l'inertie de ces roues.

- J.E. M'Connell propose un système de transport des wagons à voie normale sur des chariots en voie large.

- Robert Stephenson se montre partisan de l'écartement de 4 pieds $81 / 2$ pouces $^{22}$, mais propose, pour les voies mixtes, la pose de 4 files de rails et non de 3 . Il souligne que lors du transbordement du bétail d'un écartement à l'autre, il faut donner le temps aux bêtes de paitre et de se calmer ${ }^{23}$. Il pense que les progrès des locomotives en voie normale, notamment avec leur allongement ${ }^{24}$, donneront des performances équivalentes à celles de la voie large.

- N. Wood pense que la voie large, si elle permet de grandes vitesses, ne fait que permettre de plus grands dangers et que la vitesse de $60 m p h(100 \mathrm{~km} / \mathrm{h})$ restera une limite raisonnable que seuls franchiront

21- Pensait-il à des trains mixtes composés de matériel dans les deux écartements ?

22- On ne s'attendrait pas à moins... Son père est à l'origine de ce choix et il poursuit l'œuvre paternelle dont il a fait une grande entreprise industrielle de construction de locomotives.

23- Cette précaution écologique est-elle évoquée pour démontrer que les transbordements font perdre du temps?

24- Stephenson pense aux long boiler, mais l'expérience montra que le manque de stabilité de ces locomotives resta un handicap pour la vitesse. 
« des hommes désespérés désirant se casser le cou ». Autant rester en voie normale.

- Wyndham Harding rejette toute exploitation en voie mixte à deux écartements pour des raisons de coût et de complexité des appareils de voie : si deux voies s'embranchent sur deux autres voies dans le même écartement, il y a 6 pointes de cœur à établir, et si on réalise la même installation en double écartement, on en a 28. Les locomotives à voie large déraillent en faisant des tonneaux dans l'air (« somersaults » = roulades d'acrobate) au lieu de verser comme le font les locomotives à voie normale. Enfin des wagons pesant $4 \frac{1}{2}$ tonnes en voie large ne transportent pas plus que des wagons pesant 3 tonnes en voie normale. On peut loger 7 animaux gras (sic) dans un tombereau de 4,19 tonnes en voie large et 6 animaux dans un tombereau de 3 tonnes en voie normale.

- J.M. Laws fait remarquer que la stabilité des voitures en voie normale est telle que l'on peu transporter une tonne (sic) de bagages sur le toit de chaque voiture.

- George Bodmer, constructeur de locomotives, fait remarquer que les foyers étroits et longs produisent mieux que les foyers larges. Toutefois un écartement un peu plus large que celui de la voie normale (de 6 à 8 inches, soit environ 15 à $20 \mathrm{~cm}$ ) aurait été l'idéal.

- Edward Bury, lui aussi constructeur de locomotives et beaucoup plus connu, partage l'opinion de Bodmer sur les bienfaits d'un écartement plus large, cela pour la raison d'une plus grande liberté dans le dimensionnement des pièces des locomotives.

- John Gray, Benjamin Cubbit, William Cubbit, tous trois directeurs du matériel ou ingénieurs en chef de divers réseaux, se prononcent en faveur d'un écartement de 5 à 6 pieds pour permettre la construction de locomotives plus puissantes et, surtout, à centre de gravité beaucoup plus bas ${ }^{25}$.

- I.K. Brunel, enfin, maintient sa position en faveur de la voie de 7 pieds qu'il a préconisée pour le Great Western Railway. Brunel va même jusqu’à dire que 7 pieds est un minimum... Il pense que la coexistence

25- On se rapproche des idées de Crampton qui abaissera le centre de gravité de ses fameuses locomotives, mais démontrera, par la force des choses, que ce n'était pas la meilleure des solutions en matière de stabilité et que cela fatiguait beaucoup les voies.

26- En somme, Brunel a déjà renoncé à une position de généralisation de son écartement et admet la coexistence d'un réseau en voie large et d'autres en voie normale. 
de deux écartements en Angleterre apportera un grand bienfait en créant une émulation réciproque pour le bien de tous ${ }^{26}$. Il admet que les ruptures de charge et les transbordements resteront un inconvénient majeur.

Il semble que de ces débats résulte le constat, chez les constructeurs de locomotives, que l'écartement normal de 4 pieds $81 / 2$ pouces a été trop étroit, trop restrictif, et qu'un écartement se situant entre 5 et 6 pieds (soit entre 1500 et $1800 \mathrm{~mm}$ environ) aurait été excellent et aurait dû être imposé comme écartement standard normalisé. Mais il en résulte aussi que, chez les exploitants des réseaux, un écartement plus large aurait été d'un coût d'exploitation beaucoup plus élevé que celui de la voie normale pour des services rendus (notamment en capacité de transport) à peine plus grands. Il est certain que cette bataille des écartements, la seule véritable qu'ait connue le chemin de fer avec des positions nettes et avouées, est intervenue avec vingt années de retard. Les Anglais se heurteront à une situation analogue avec leur gabarit, mais pourront faire profiter les réseaux du reste du monde de leur expérience en leur évitant le choix d'un gabarit aussi restrictif. Les réseaux américains, en particulier, sauront jouer au maximum de cette carte. 